\title{
Unusual presentation of tropical pulmonary eosinophilia
}

\author{
VK JAIN, OP BENIWAL \\ From the Department of Tuberculosis and Chest Diseases, Sardar Patel Medical College, Bikaner, India
}

Typical clinical and radiological features of tropical pulmonary eosinophilia were originally described by Weingarten. ${ }^{\prime}$ Cases of tropical pulmonary eosinophilia were initially recorded in India, but the disease is now known to occur throughout the world. Various atypical radiological presentations such as cavitation, ${ }^{2-4}$ pleural effusion, ${ }^{34}$ and patchy pneumonitis ${ }^{5-1}$ have been reported. Very few cases with a large area of pneumonitis ${ }^{48}$ have been recorded. In view of its rarity we present a case of tropical pulmonary eosinophilia in which a large area of pneumonia like consolidation was present in the right lower zone.

\section{Case report}

A young Hindu man aged 16 years, who did not smoke or drink, presented with symptoms of cough with scanty sputum and breathlessness on exertion of six months' duration and pain in the chest of 15 days' duration. During this period there was no significant history of fever, loss of appetite, haemoptysis or worm infestation. Six months previously he had consulted a general practitioner, who diagnosed pulmonary tuberculosis on the basis of the symptoms and radiographical evidence of a large area of consolidation in the right lower zone with small patchy areas of opacification in the left lower zone. He was given antituberculous treatment-a combination of isoniazid and ethambutol. The patient took the drugs regularly until he was admitted to this hospital. He showed neither symptomatic relief nor radiological improvement during this period.

General examination revealed nothing important. Examination of the respiratory system showed diminished movement, dullness to percussion, and diminished air entry with fine crackles at the right base without evidence of mediastinal shift. A few fine crackles were also heard over left base. The total leucocyte count was $21 \times 10^{9} / 1$ (polymorphs $50 \%$, lymphocytes $22 \%$, eosinophils $28 \%$ ); an absolute eosinophil count was $3.25 \times 10^{\circ} /$. The erythrocyte sedimentation rate (ESR) was $84 \mathrm{~mm}$ in one hour by the Westergren method. Urine and stool examination showed no abnormality. Examination of sputum smears for acid fast bacilli repeatedly gave negative results. Sputum smears were also negative for malignant cells. A tuberculin test using 1 tuberculin unit PPD-RT 23 with Tween 80 gave a negative response. Sputum smears and cultures were negative for fungi and pyogenic organisms. The chest

Address for reprint requests: Dr OP Beniwal, Department of Tuberculosis and Chest Diseases, A-2 PBM Hospital Campus, Bikaner 334001, India.

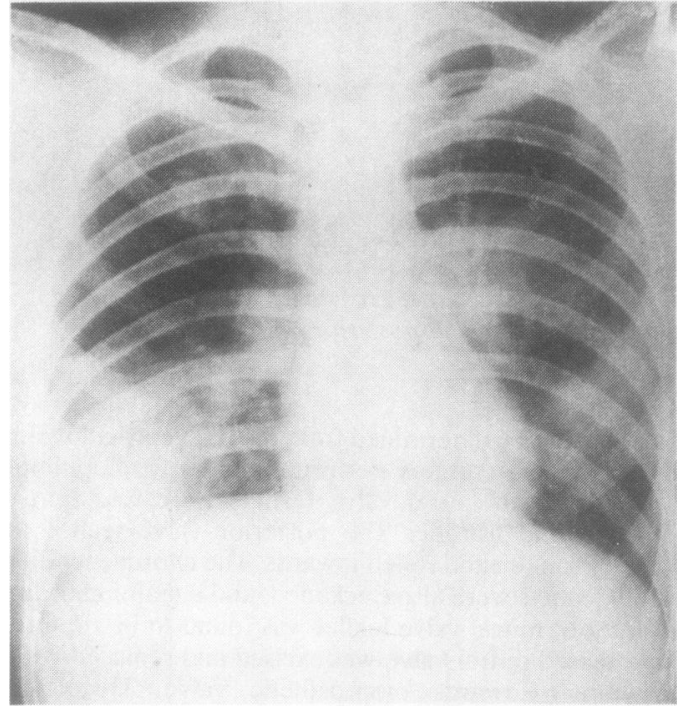

Fig 1 Chest radiograph showing a large area of consolidation in the right lower zone with small patchy areas in the left lower zone.

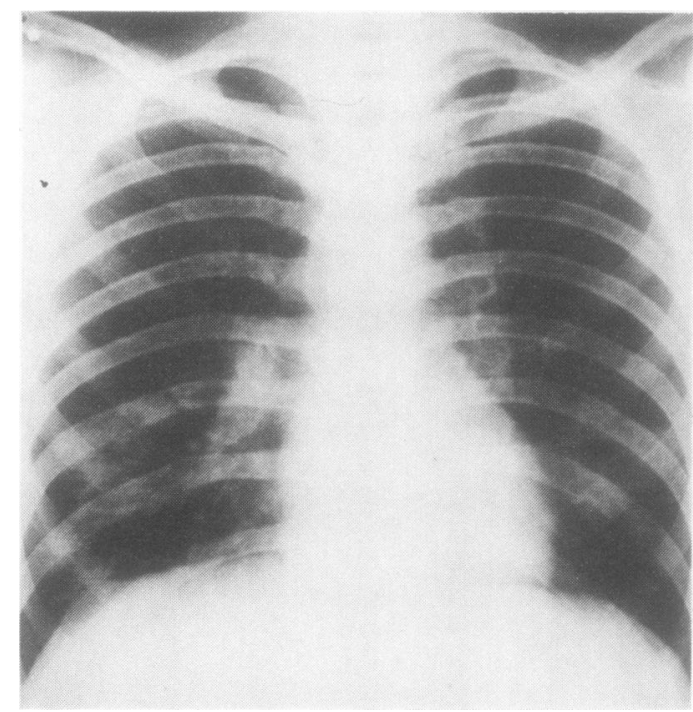

Fig 2 Chest radiograph after three weeks of treatment showing clearing. 
radiograph (fig 1) showed evidence of a large area of consolidation in the right lower zone with small patchy areas of opacification in the left lower zone. The patient was treated with $450 \mathrm{mg}$ of diethyl carbamazine orally in three divided doses daily for three weeks. A chest radiograph (fig 2) taken after three weeks of treatment showed appreciable clearing of the shadows. During this period no antibiotics or antituberculous drugs were prescribed. At the end of treatment the total leucocyte count was $9.5 \times 10^{9} / 1$ (polymorphs $64 \%$, lymphocytes $29 \%$, eosinophils $7 \%$ ) and the absolute eosinophil count $0.6 \times 10^{9} / 1$. The ESR was $30 \mathrm{~mm}$ in one hour.

\section{Discussion}

Radiological presentation of tropical pulmonary eosinophilia as a large area of pneumonic consolidation is rare. Nath and Jain reported a patient with a large area of pneumonia at right base associated with increased peripheral blood eosinophil counts. The shadow disappeared completely after three weeks of diethyl carbamazine treatment. Chaudhary $e t a^{8}$ also reported an unusual case of tropical pulmonary eosinophilia presenting with an area of pneumonic consolidation $3-4 \mathrm{~cm}$ in diameter in the right mid zone with increased peripheral blood eosinophil counts. This patient responded to three weeks' treatment with diethyl carbamazine. Our patient was initially misdiagnosed as having pulmonary tuberculosis but showed no response to antituberculous treatment. The radiological shadow in the right lower zone could have been due to either pneumonia, lung abscess, malignancy, or lower lobe tuberculosis. After scrutiny of the blood film the patient was diagnosed as suffering from tropical pulmonary eosinophilia and this diagnosis was supported by complete radiological, haematological, and symptomatic response to diethyl carbamazine without the addition of any antibiotics.

\section{References}

' Weingarten RJ. Tropical eosinophilia. Lancet 1943;i:103-5.

${ }^{2}$ Menon NK. Tropical eosinophilia-atypical manifestations. Indian J Chest Dis 1963;5:231-6.

${ }^{3}$ Viswanathan R. Pulmonary eosinophiliosis. $Q \quad J$ Med 1948; 17:257-70.

${ }^{4}$ Nath J, Jain VK. Atypical presentation of pulmonary eosinophilia. Indian J Chest Dis Allied Sci 1978;20:141-4.

${ }^{5}$ Mital OP, Someswar Rao M, Prasad R, et al. Tropical pulmonary eosinophilia: unusual presentation. Indian $J$ Chest Dis 1975;17:135.

${ }^{6}$ Herlinger H. Pulmonary changes in tropical eosinophilia. $\mathrm{Br} J$ Radiol 1963;36:889-901.

' Khoo FY, Dhan Raj TJ. The roentgenographical appearance of eosinophilic lung (tropical eosinophilia). Am J Roentgenol 1960;83:251.

${ }^{8}$ Chaudhary BS, Gupta PK, Gupta PR. Tropical pulmonary eosinophilia: an unusual presentation. Indian J Chest Dis Allied Sci 1978;20:139-40. 